Supporting Information

\title{
A Thermostable Protein Matrix for Spectroscopic Analysis of Organic Semiconductors
}

George A. Sutherland, Daniel Polak, David J. K. Swainsbury, Shuangqing Wang, Frank C. Spano, Dirk B. Auman, David G. Bossanyi, James P. Pidgeon, Andrew Hitchcock, Andrew J. Musser, John E. Anthony, P. Leslie Dutton, Jenny Clark and C. Neil Hunter*

Table of Contents

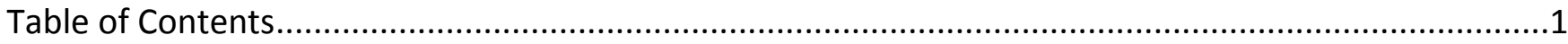

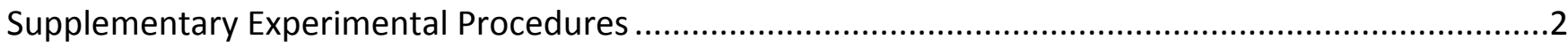

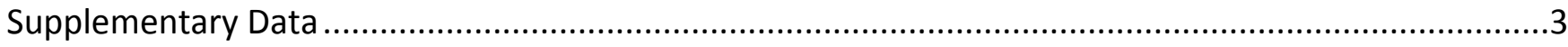

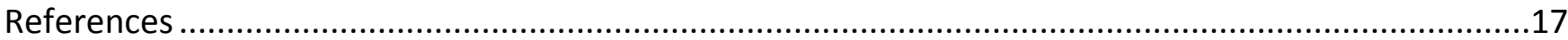




\section{Supplementary Experimental Procedures}

Protein Purification: Proteins were produced and purified essentially as described previously. ${ }^{1}$ Briefly, genes encoding BT6 and $4 \mathrm{~A}$ cloned into pJexpress414 and proteins produced in semi-aerobic cultures of BL21(DE3) E. coli by isopropyl $\beta$-D-1thiogalactopyranoside induction $(1 \mathrm{mM})$ at $18{ }^{\circ} \mathrm{C}$ for $16 \mathrm{~h}$. Cells were harvested, lysed by sonication on ice and purified by immobilized metal affinity chromatography. Proteins were further purified by size exclusion chromatography on a Superdex 200 increase column (GE Healthcare). Protein purity was assessed by SDS-PAGE.

OSC Attachment: All buffers and solvents were sparged with nitrogen gas for 30 min prior to use, with the chromophores kept strictly in the dark. AXT stocks $\left(1 \mathrm{mg} \mathrm{mL}^{-1}\right)$ were prepared in $100 \%$ DMSO and mixed 1:4 with protein solutions in 50 mM CHES pH 10. A 10-fold molar excess of AXT (336 $\mu \mathrm{M})$ over protein (33.6 $\mu \mathrm{M})$ was established in all experiments. The samples were then diluted 8-fold in $50 \mathrm{mM} \mathrm{CHES} \mathrm{(} \mathrm{pH} 10)$, filtered (0.2 $\mu \mathrm{m}$ pore diameter; Sartorius) and applied to a $5 \mathrm{~mL}$ DEAE sepharose anion exchange column. Protein was eluted with $50 \mathrm{mM} \mathrm{HEPES,} 500 \mathrm{mM} \mathrm{NaCl}(\mathrm{pH} 6)$. Incorporation of ECH and $\beta C$ was achieved using an identical method to that described above, but with both carotenoid stocks prepared at $30 \mu \mathrm{g}$ $\mathrm{mL}^{-1}$ in DMSO and protein concentrations adjusted accordingly to maintain a 10-fold pigment excess. Solutions of Rubr (1 mg $\left.\mathrm{mL}^{-1}\right)$, EP-PBI $\left(1 \mathrm{mg} \mathrm{mL}^{-1}\right)$, C8BTBT $\left(0.2 \mathrm{mg} \mathrm{mL}^{-1}\right)$, and T-Tet $\left(0.25 \mathrm{mg} \mathrm{mL}^{-1}\right)$ were made up in DMSO following heating $\left(80{ }^{\circ} \mathrm{C}\right)$ and sonication. Stock solutions were mixed 2:5 with $50 \mathrm{mM} \mathrm{CHES} \mathrm{(pH} \mathrm{10)} \mathrm{containing} 4 \mathrm{~A}(40 \mu \mathrm{M})$, filtered and buffer exchanged (8000-fold effective dilution) into $50 \mathrm{mM}$ HEPES (pH 6).

Trehalose Glass Formation: 4A-OSC samples were mixed 1:1 with an aqueous solution of $0.5 \mathrm{M}$ trehalose, $0.5 \mathrm{M}$ sucrose and drop-cast onto quartz-coated glass (Ossila). The mixtures were then desiccated under vacuum (-70 kPa, Pelco Hot Vac) for 4 days at room temperature with a large excess of calcium sulfate (Drierite).

Cryo-EM: Cryo-TEM grids were prepared using a Leica GP2 plunge freezer system. Briefly, $5 \mu$ protein samples were incubated on freshly glow-discharged quantifoil 1.2/1.3 400 mesh carbon coated copper grids for $5 \mathrm{~s}$ at $25^{\circ} \mathrm{C}$ and a relative humidity of $80 \%$, blotted on Whatman Grade 1 filter paper for $3 \mathrm{~s}$ and immediately plunge frozen in liquid ethane at $-176^{\circ} \mathrm{C}$. Grids were imaged at 39000x magnification on a Techni Arctica TEM with a spot-size of 2 exposing each image for $2 \mathrm{~s}$.

Transient Absorption Spectroscopy: Sub-picosecond transient absorption (TA) measurements were recorded on a modified Helios Fire Commercial TA system (Ultrafast Systems) with a set up similar in function to experimental systems previously described. ${ }^{2}$ Briefly, narrow excitation pulses ( $10 \mathrm{~nm}$ bandwidth) were generated with a $\beta$-barium borate crystal by doubling the fundamental $(800 \mathrm{~nm})$ from a $10 \mathrm{kHz}$ regenerative amplifier giving $400 \mathrm{~nm}$ (3.09 eV) excitation (Solstice, Spectra-Physics). Pump fluences were limited to below $4 \times 10^{14}$ photon per pulse per $\mathrm{cm}^{2}$ to avoid degradation of samples. The $800 \mathrm{~nm}$ output from the regenerative amplifier was passed through a sapphire crystal to generate white light, which was used as the probe pulse. The transmitted probe beam was then dispersed by a reflective grating and detected by a silicon detector (Ultrafast Systems). The differential transmission $(\Delta T / T)$ in the sample was recorded as a function of probe photon energy and pulseprobe delay. The experimental setup enabled a temporal resolution of approximately $200 \mathrm{fs}$. 


\section{Supplementary Data}

Table S1. Amino acid sequences and physical properties of proteins used in this study.

\begin{tabular}{|c|c|c|c|c|}
\hline \multirow[t]{2}{*}{ Protein } & \multirow[t]{2}{*}{ Sequence ${ }^{[\mathrm{a}]}$} & \multicolumn{3}{|l|}{$\mathrm{T}_{\mathrm{m}}\left({ }^{\circ} \mathrm{C}\right)$} \\
\hline & & $\mathrm{pH} 6$ & $\mathrm{pH} 7.5$ & $\mathrm{pH} 9$ \\
\hline BT6 & $\begin{array}{l}\text { MGGDGENLYFQG } \\
\text { EIWKQHEDALQKFEEALNQFEDLKQLGGSGSGSGG } \\
\text { EIWKQHEDALQKFEEALNQFEDLKQLGGSGSGSGG } \\
\text { EIWKQHEDALQKFEEALNQFEDLKQLGGSGSGSGG } \\
\text { EIWKQHEDALQKFEEALNQFEDLKQLHHHHHH }\end{array}$ & 79 & 37 & 34 \\
\hline $4 \mathrm{~A}$ & $\begin{array}{l}\text { MGGDGENLYFQG } \\
\text { EIWKQAEDALQKFEEALNQFEDLKQLGGSGSGSGG } \\
\text { EIWKQAEDALQKFEEALNQFEDLKQLGGSGSGSGG } \\
\text { EIWKQAEDALQKFEEALNQFEDLKQLGGSGSGSGG } \\
\text { EIWKQAEDALQKFEEALNQFEDLKQLHHHHHH }\end{array}$ & $>90^{[b]}$ & 52 & 49 \\
\hline
\end{tabular}

[a] The positions of residue substitutions are shown in bold. [b] The midpoint of the melting curve could not be accurately determined within temperature range tested; sample displayed typically helical signal at $90^{\circ} \mathrm{C}$. 


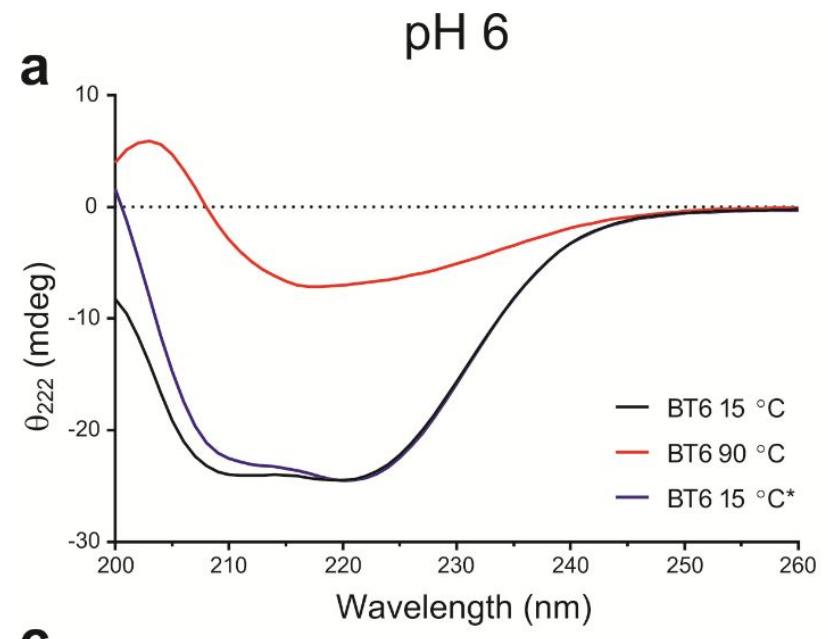

C
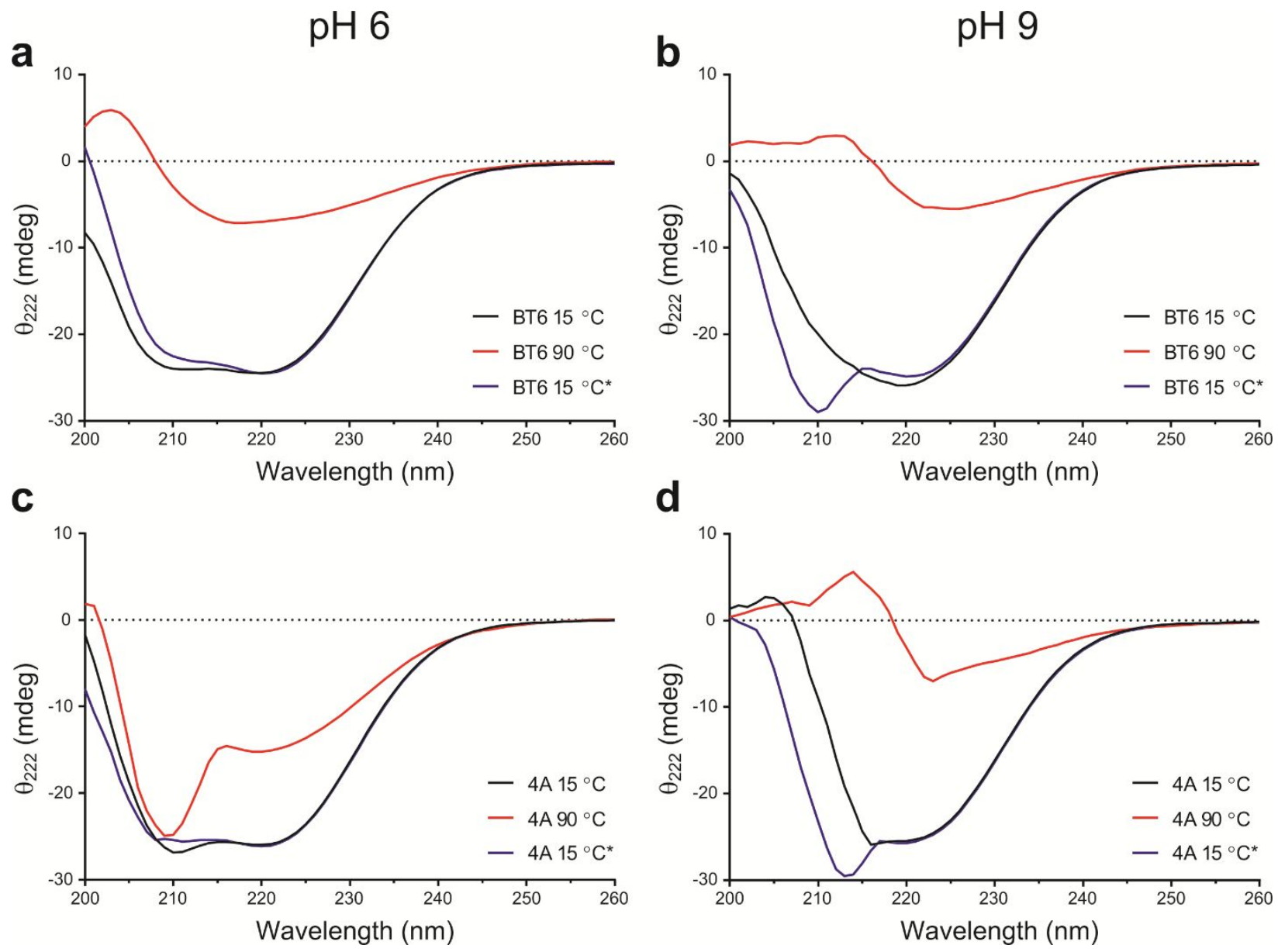

Figure S1. Circular dichroism spectroscopy of apo-maquette proteins. Spectra of BT6 (a, b) and $4 \mathrm{~A}\left(\mathrm{c}\right.$, d) are shown at $15{ }^{\circ} \mathrm{C}$ (black), $90{ }^{\circ} \mathrm{C}$ (red) and following cooling to $15^{\circ} \mathrm{C}$ (blue). Spectra were recorded at pH 6 (left) and pH 9 (right). 
a

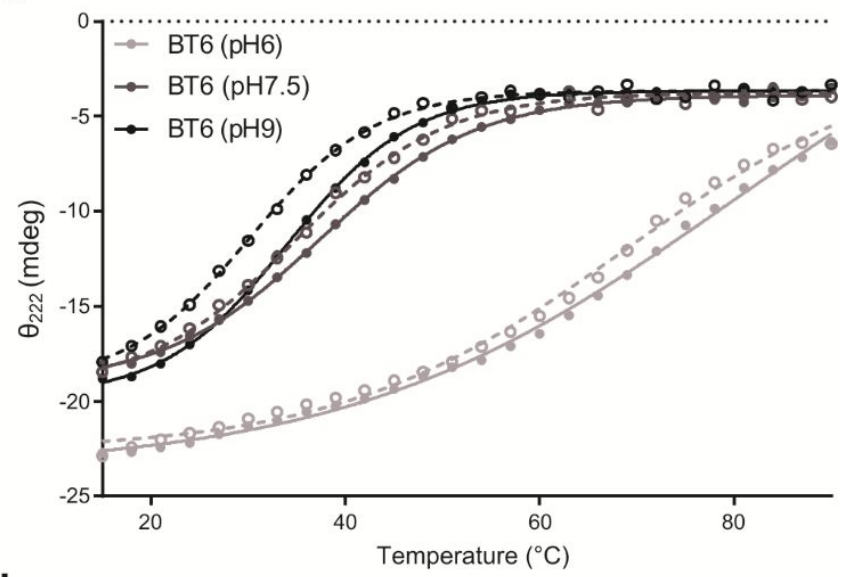

b

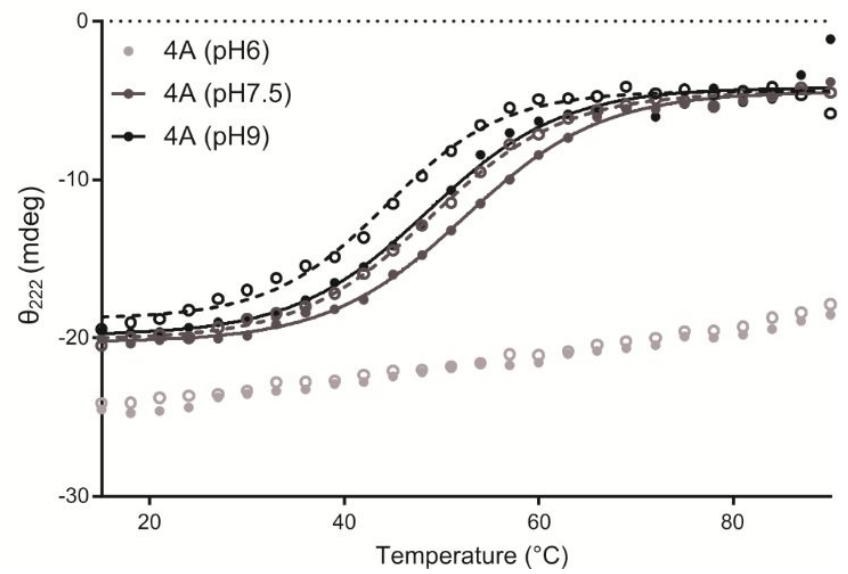

Figure S2. Thermal stability of maquette proteins. Melting (solid markers) and refolding (open markers) of BT6 (a) and 4A (b) in aqueous buffer at the indicated $\mathrm{pH}$. Ellipticity was recorded every $1^{\circ} \mathrm{C}$ but only every third data point is shown for clarity. Where possible, data points are described by a Boltzmann distribution for melting (solid lines) or refolding (dashed lines). 


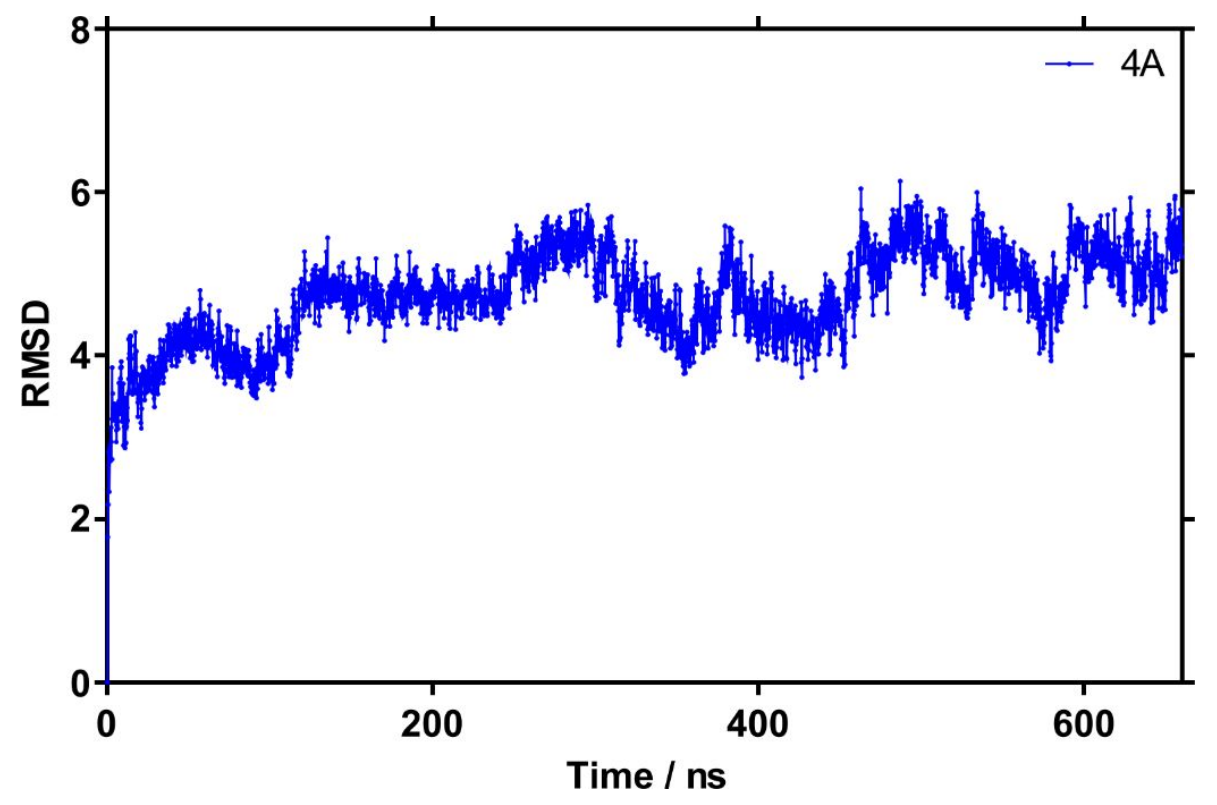

Figure S3. Root mean squared deviation (RMSD) values for the 4A protein structure during a 660 ns simulated trajectory. 


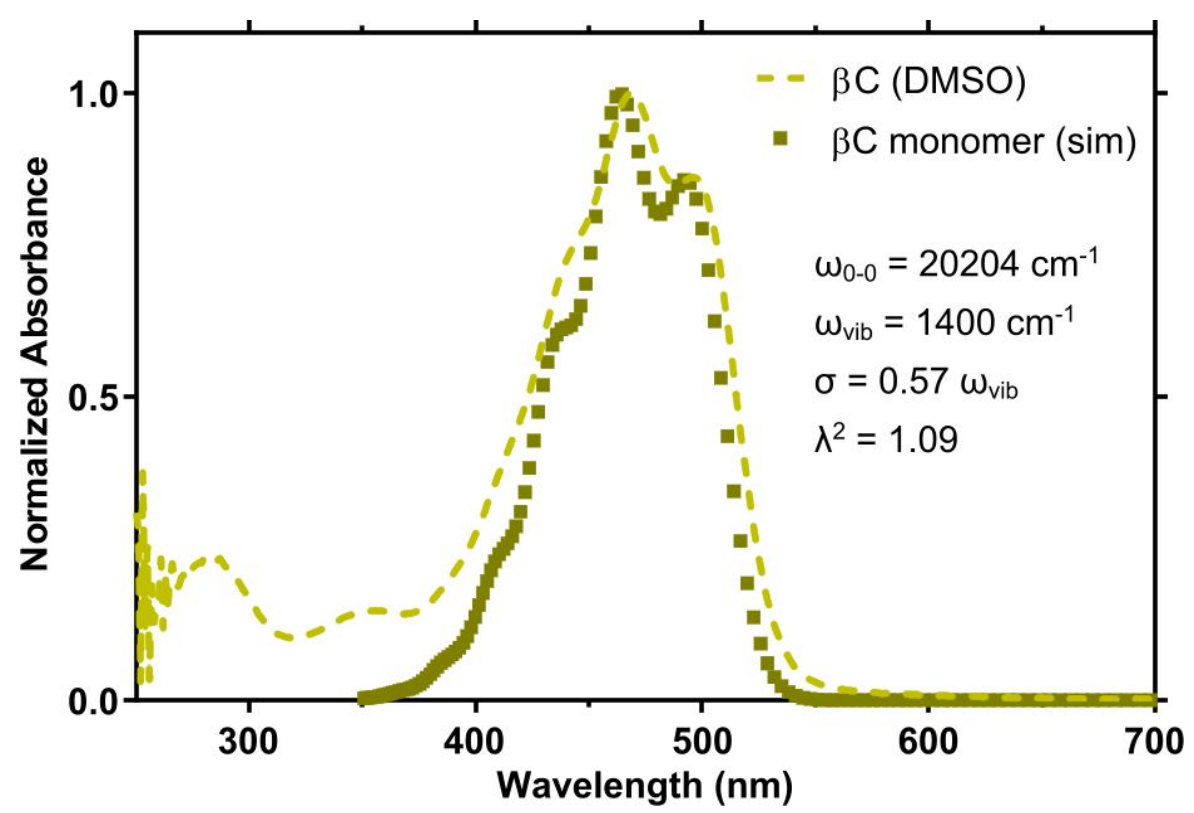

Figure S4. Simulated absorption spectra of monomeric $\beta C$ (dark orange squares) compared to experimental values (yellow, dashed line). Values corresponding to the 0-0 energy $\left(\omega_{0-0}\right)$, the vibrational energy most strongly coupled to the S0-S2 transition $\left(\omega_{\text {vib }}\right)$, the linewidth of the transitions $(\sigma)$ and the Huang-Rhys parameter $\left(\lambda^{2}\right)$ used in the simulation are quoted (inset). 
(a) Dimer (varying $\varphi$ ) $\vee_{\mathrm{nn}}=1400 \mathrm{~cm}^{-1}$

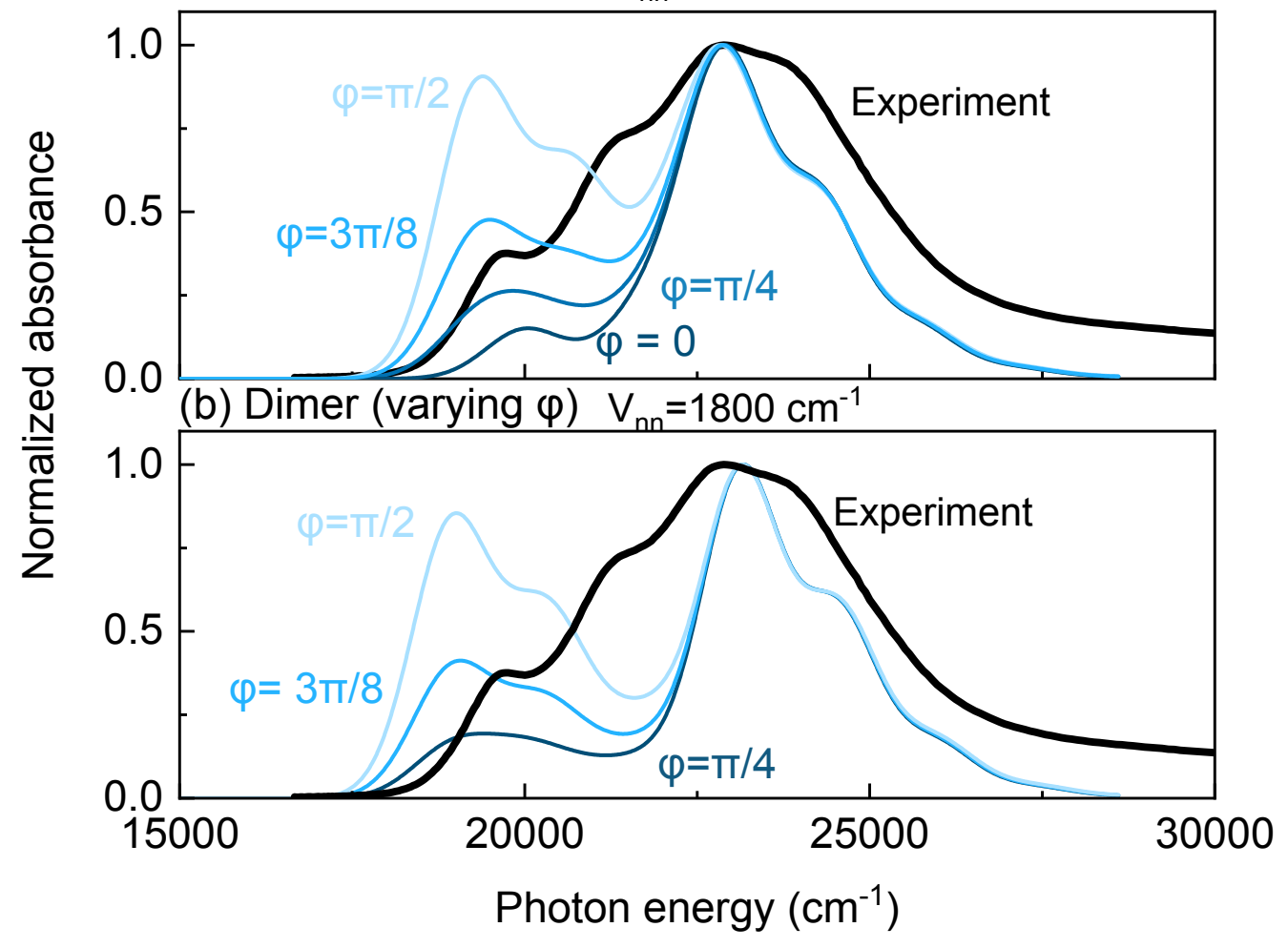

Figure S5. Modeling the absorption spectrum of dimeric $\beta C$. Experimental absorption spectrum of the $4 A-\beta C$ complex (black) and simulated absorption spectra (blue traces) of a dimer using the Holstein Hamiltonian parameterized using $\omega_{\text {vib, }} \lambda^{2}$ and $\omega_{0-0}$ from Figure S4 and the nearest neighbor coupling $\left(V_{n n}\right)$ shown in the figure insets. The Hamiltonian was represented in a basis set consisting of all one- and two-particle states with the total number of vibrational quanta capped at 6 . $\phi$ is the angle between transition dipole moments on neighboring molecules. 
(a) Trimer (varying $\varphi$ )

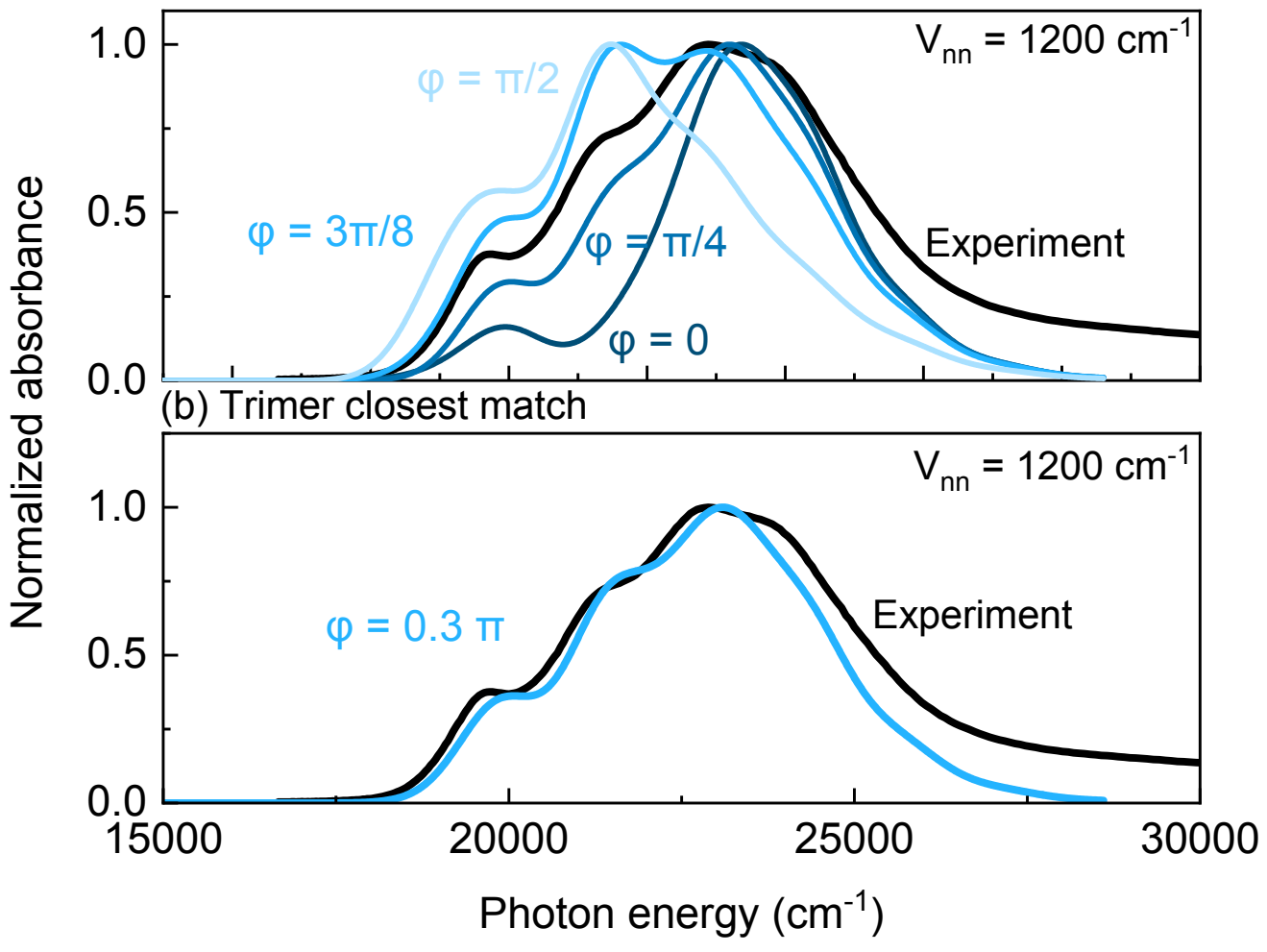

Figure S6. Modeling the absorption spectrum of a $\beta C$ trimer. Experimental absorption spectrum of $4 A-\beta C$ (black) and simulated absorption spectra of a linear trimer (blue traces) using the Holstein Hamiltonian as described in Figure S4 and employing the two-particle approximation. $\phi$ is the angle between transition dipole moments on neighboring molecules hence the aggregates are chiral - and $V_{n n}$ is the nearest-neighbor coupling. 
(a) Tetramer (varying $\varphi, V_{\mathrm{nn}}=1200 \mathrm{~cm}^{-1}$ )
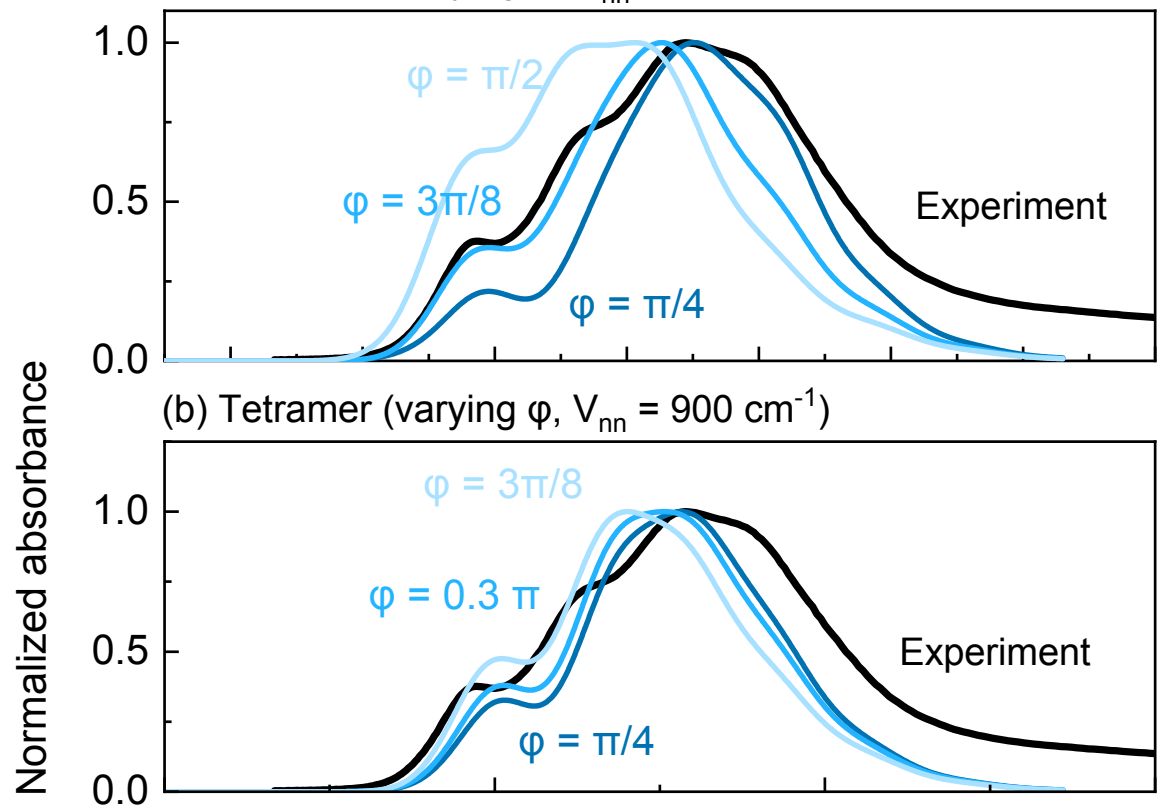

(c) Decamer (varying $\varphi, V_{\mathrm{nn}}=1200 \mathrm{~cm}^{-1}$ )

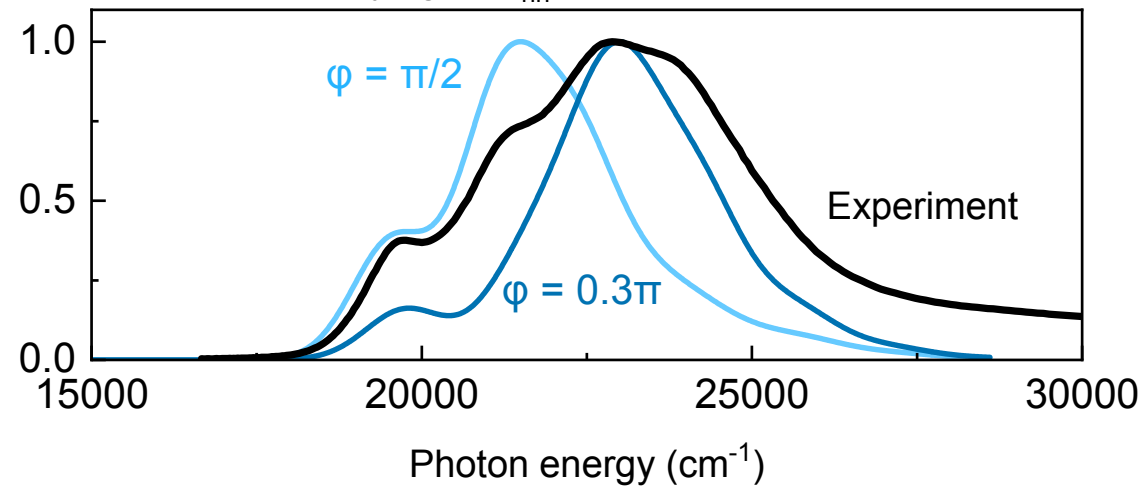

Figure S7. Modeling the absorption spectrum of larger $\beta C$ ensembles. Experimental absorption spectrum of $4 A-\beta C$ (black) and simulated absorption spectra of a $\beta C$ tetramer $(\mathbf{a}, \mathbf{b})$ and decamer $(\mathbf{c})$ using the Holstein Hamiltonian described in Figure S4 and using the two-particle approximation. All aggregates are linear (open boundary conditions) and chiral, with $\phi$ being the angle between transition dipole moments on neighboring molecules. $\mathrm{V}_{\mathrm{nn}}$ is the nearest-neighbor coupling. 

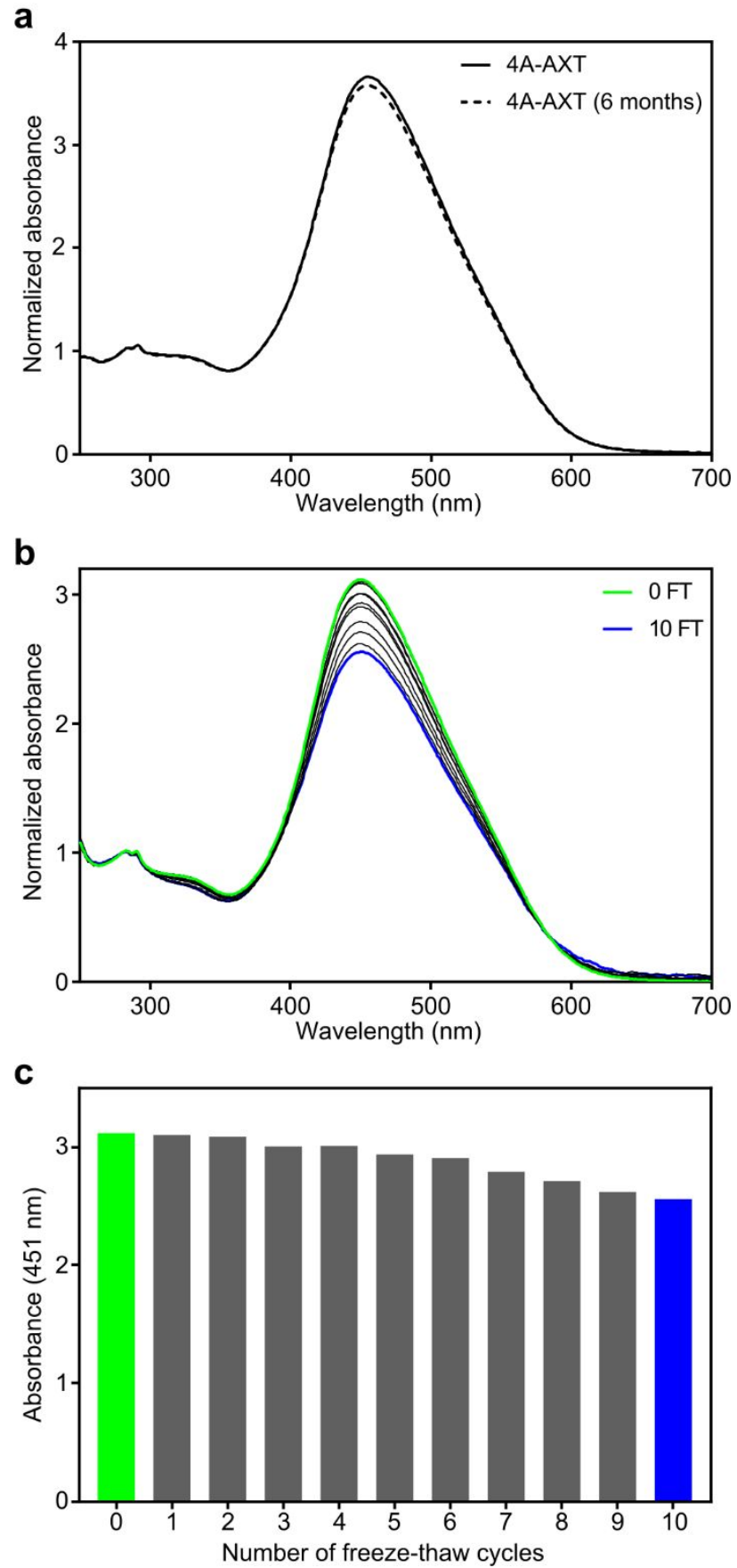

Figure S8. Long term storage of the 4A-AXT complex. a Absorption spectra of a freshly generated 4A-AXT sample (solid line) and the same sample following storage for 6 months at $-80{ }^{\circ} \mathrm{C}$ (dashed line); normalized to tryptophan absorbance at 280 $\mathrm{nm}$. b Absorption spectra of the same 4A-AXT sample following successive rounds of freezing and thawing (FT) with the original sample (green) and the sample following 10 rounds of freeze-thawing (blue) highlighted. Intervening samples are shown as narrow black lines, all samples were normalized to tryptophan absorbance at $280 \mathrm{~nm}$. c $451 \mathrm{~nm}$ absorbance from panel b displayed for all samples. 


\section{$93 \mu \mathrm{M}$}
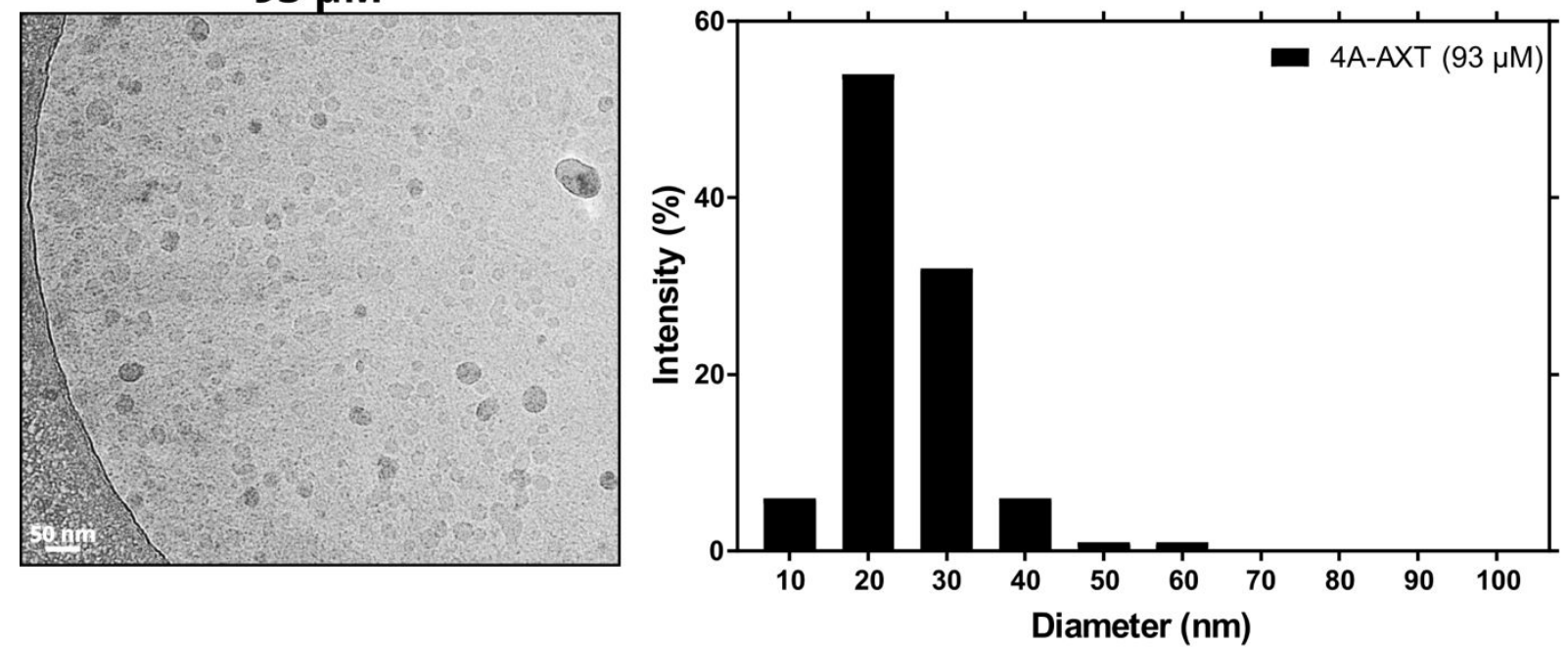

$23 \mu \mathrm{M}$
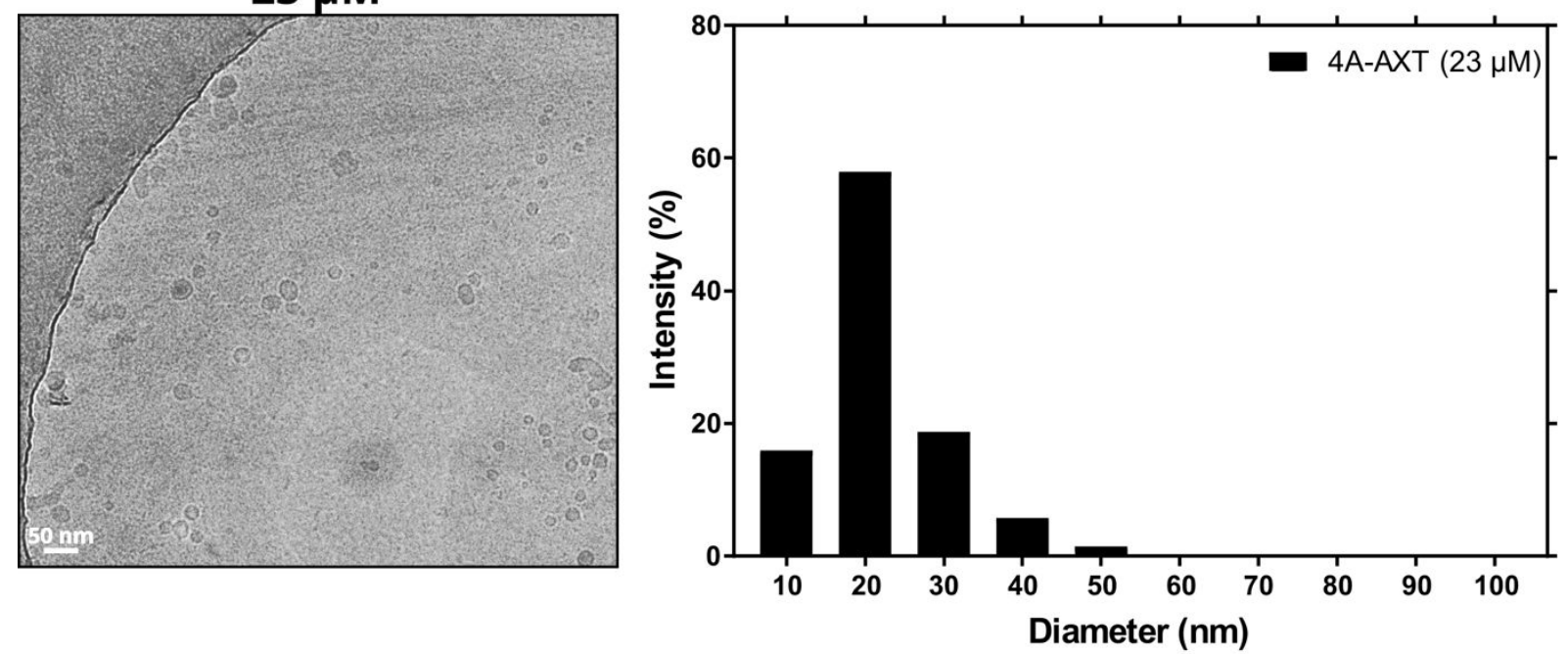

$12 \mu \mathrm{M}$
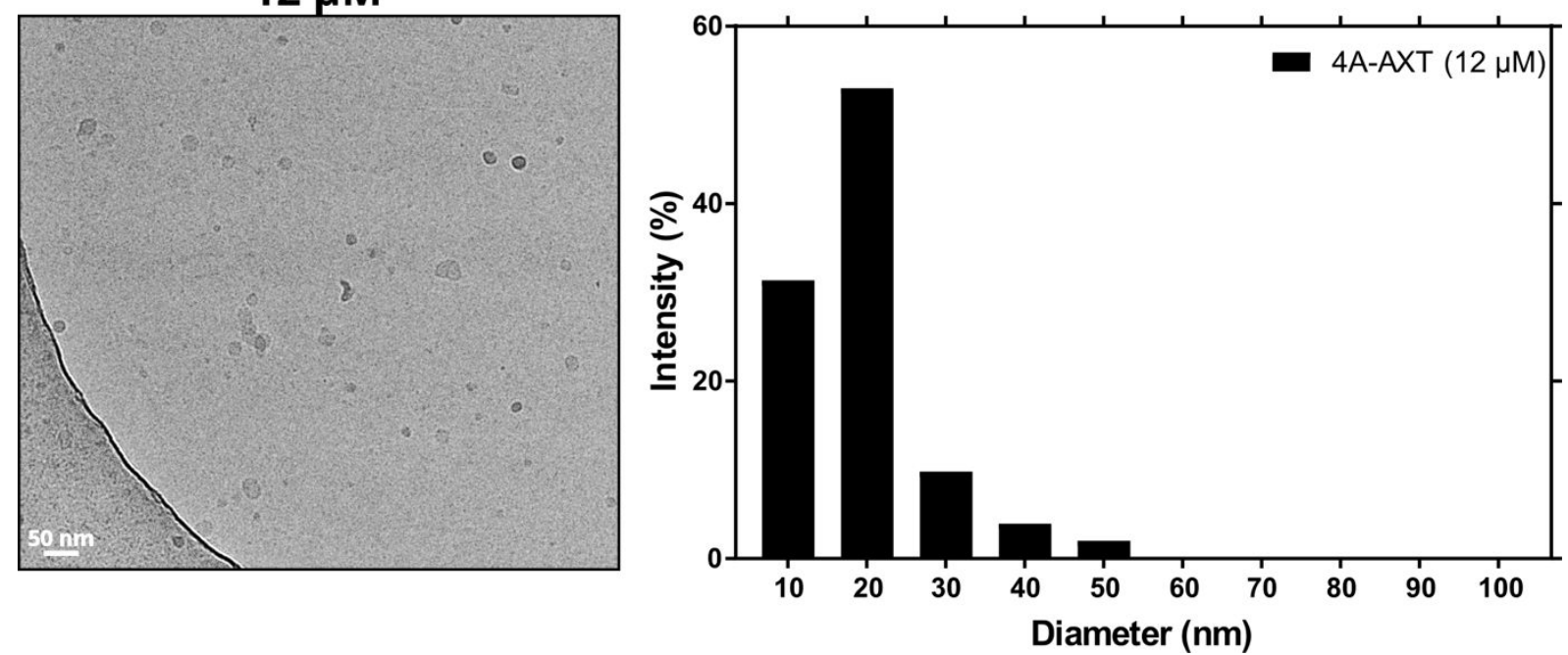

Figure S9. Morphology of 4A-AXT. Cryo-electron microscopy (left) of 4A-AXT at various concentrations. Images representative of at least 3 experimental repeats, with $>5$ grid squares assessed for each sample. Particle diameters were measured using ImageJ $(\mathrm{NIH})$ and plotted according to frequency. All particles were measured where a full structure could be resolved, apart from at $93 \mu \mathrm{M}$ where 100 measurements were taken. Protein concentrations calculated using a Bradford assay according to the manufacturer's instructions. 


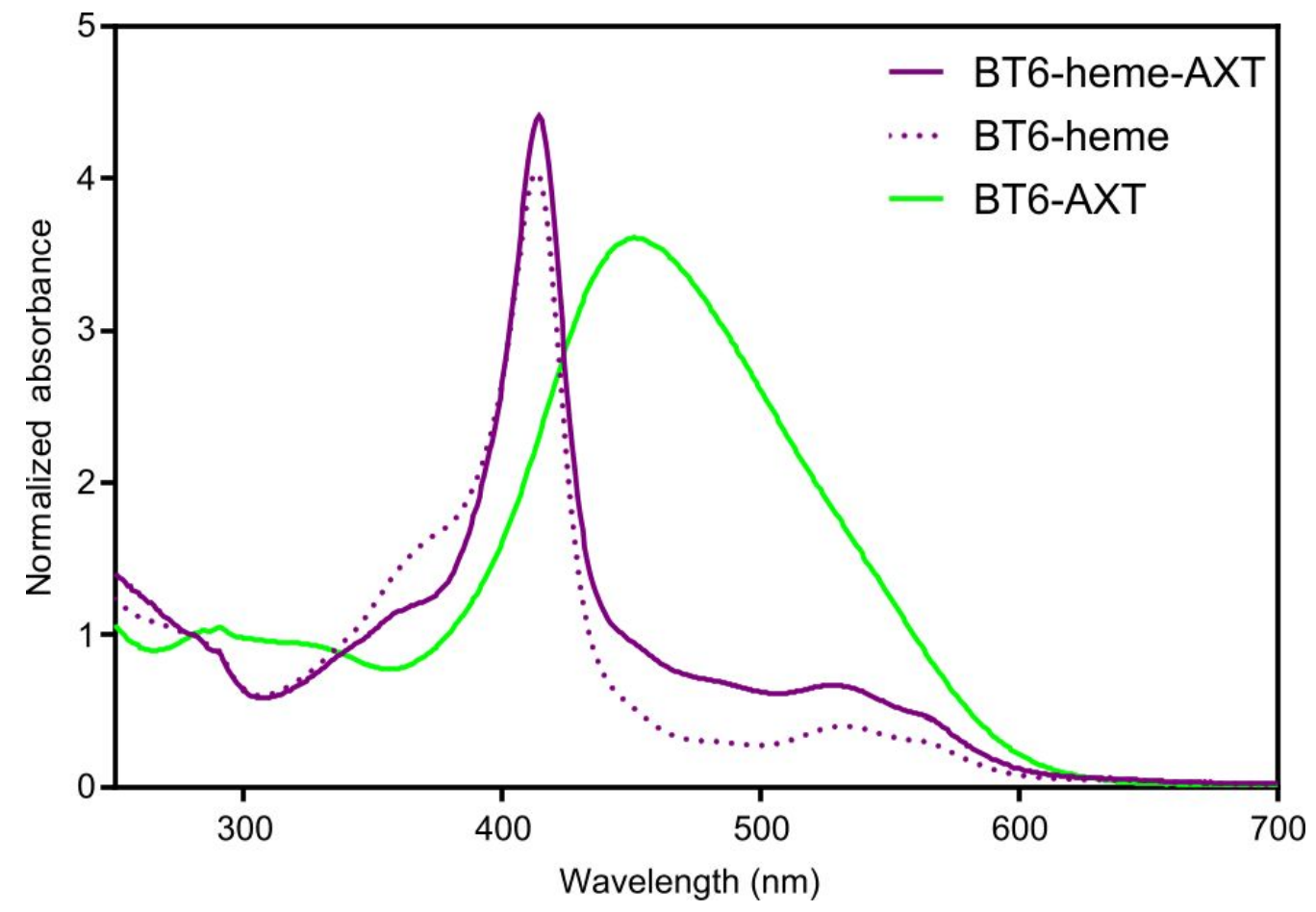

Figure S10. Absorption spectra of heme-bound BT6 (purple, dotted line) and BT6-heme following reconstitution with AXT (purple, solid line). AXT-bound BT6 is shown for reference (green, solid line). All spectra normalized to tryptophan absorbance at $280 \mathrm{~nm}$. 

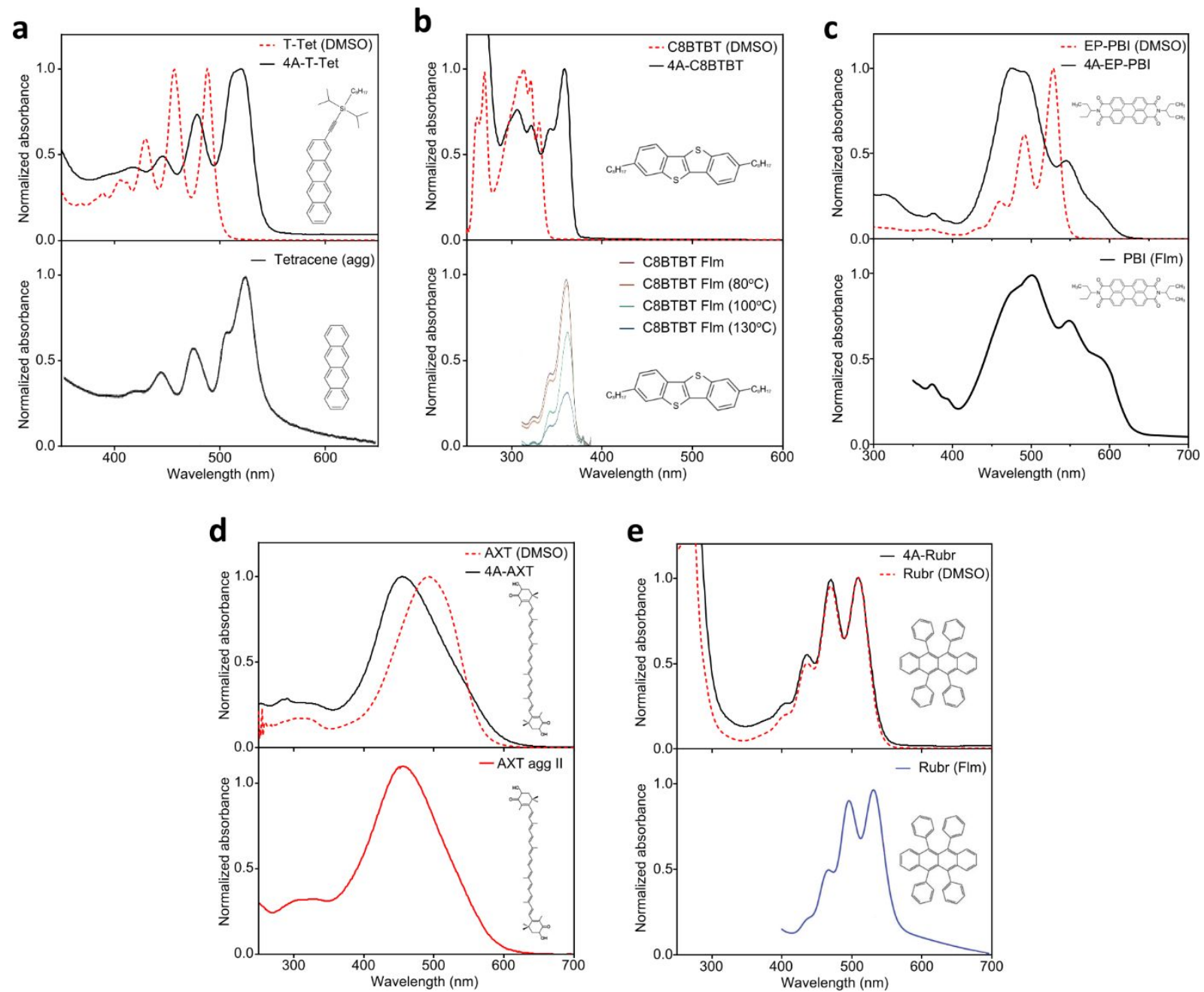

Figure S11. a-e Normalized absorption spectra of various OSCs (upper graphs) as monomer in solvent (dashed red lines) and in protein-complexes (black lines). All spectra are compared with previously reported solution aggregates or thin-films (Flm) of structurally similar molecules, which are reproduced with permissions to enable comparison (lower graphs). Chemica structures of the OSCs used are shown in each graph (inset). (a) Reproduced with permission from Kim et al. ${ }^{3}$ (C) 2003, American Chemical Society. (b) Reproduced with permission from Keum et al. ${ }^{4}$ (C) 2018, Springer Nature. (c) Reproduced with permission from Kennehan et al. ${ }^{5}$ (C) 2017, Royal Society of Chemistry. (d) Reproduced with permission from Musser et al.2 (C) 2015, American Chemical Society. (e) Reproduced with permission from Hodges et al. ${ }^{6}$ C 2017 , Wiley-VCH Verlag GmbH \& Co. 
a

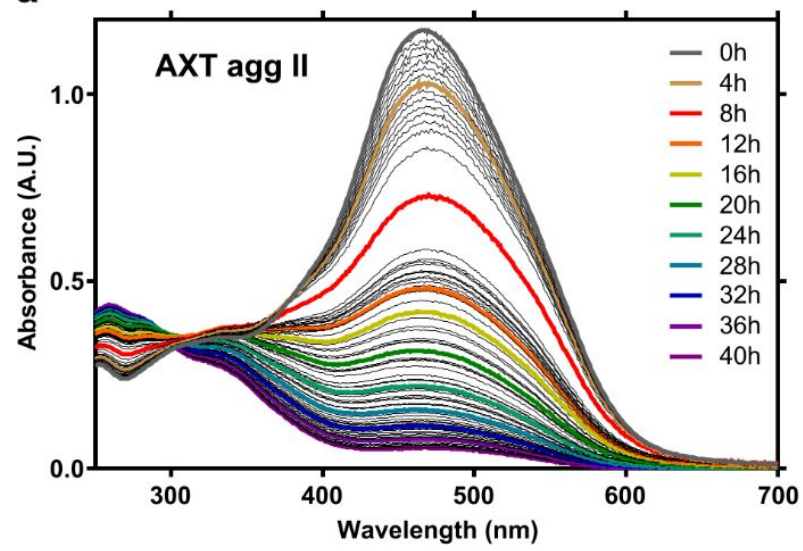

b

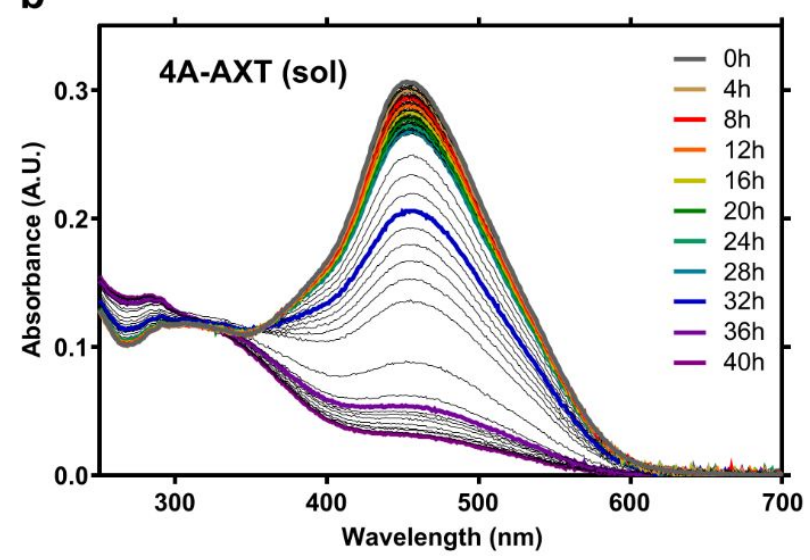

C

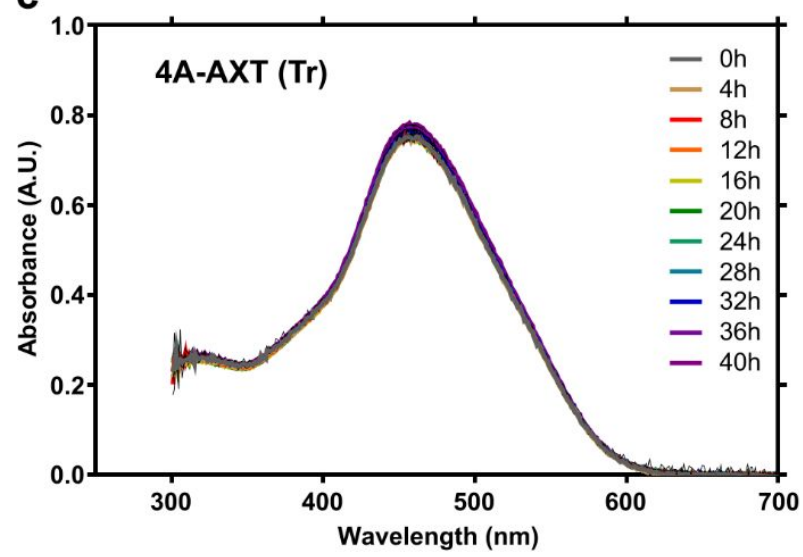

d

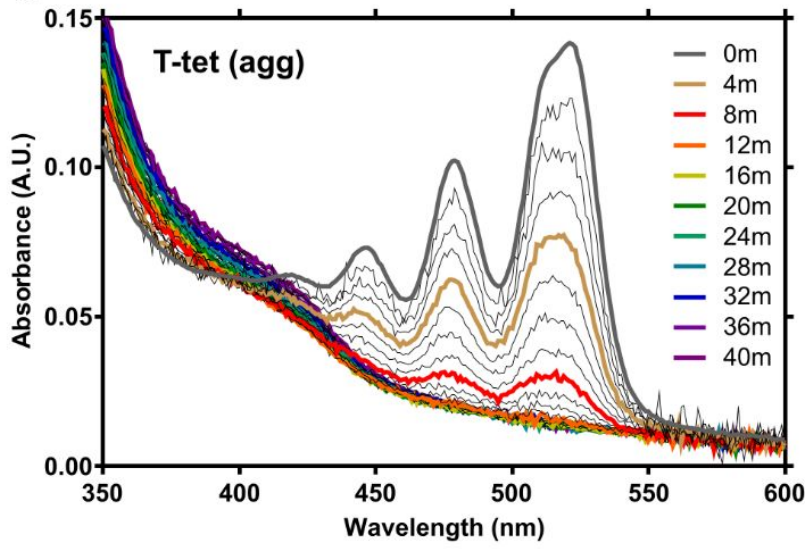

e

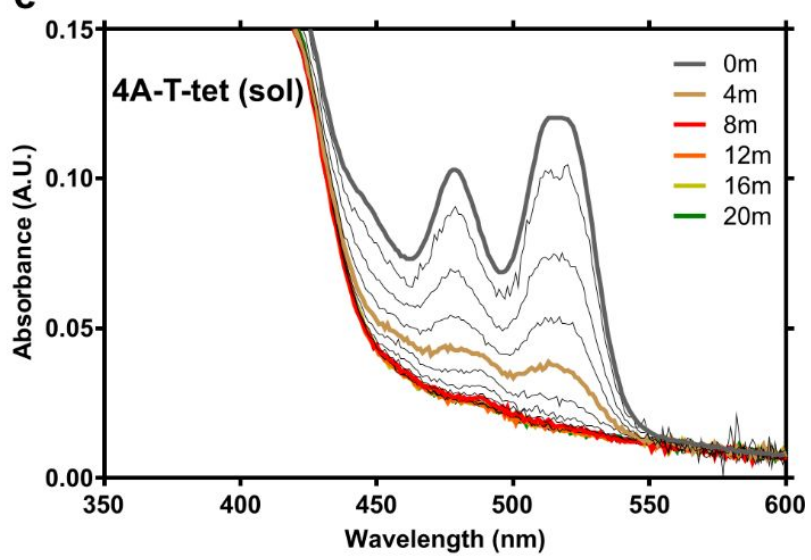

f

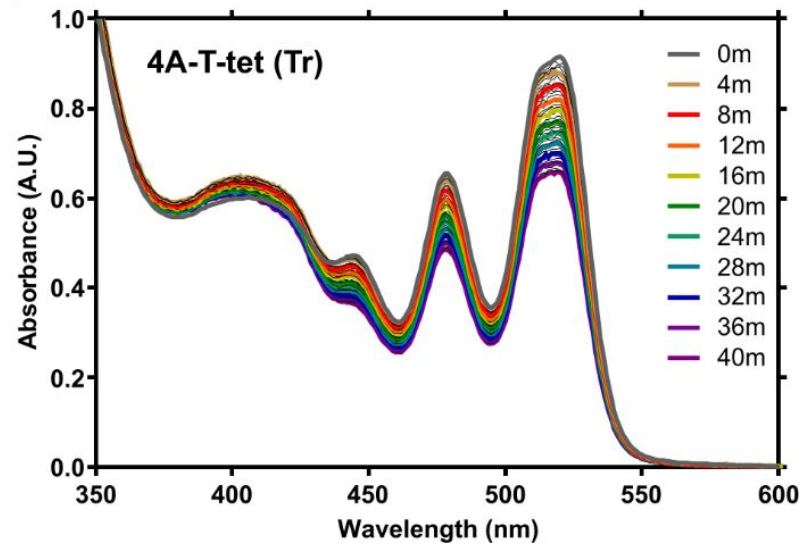

Figure S12. Photostability of AXT (a-c) and T-Tet (d-f). Spectra of solution aggregates (a, d), 4A-nanoparticles (b, e) and trehalose glasses (c, f) were recorded every $30 \mathrm{~min}$ for AXT and every $1 \mathrm{~min}$ for T-Tet under constant illumination. 

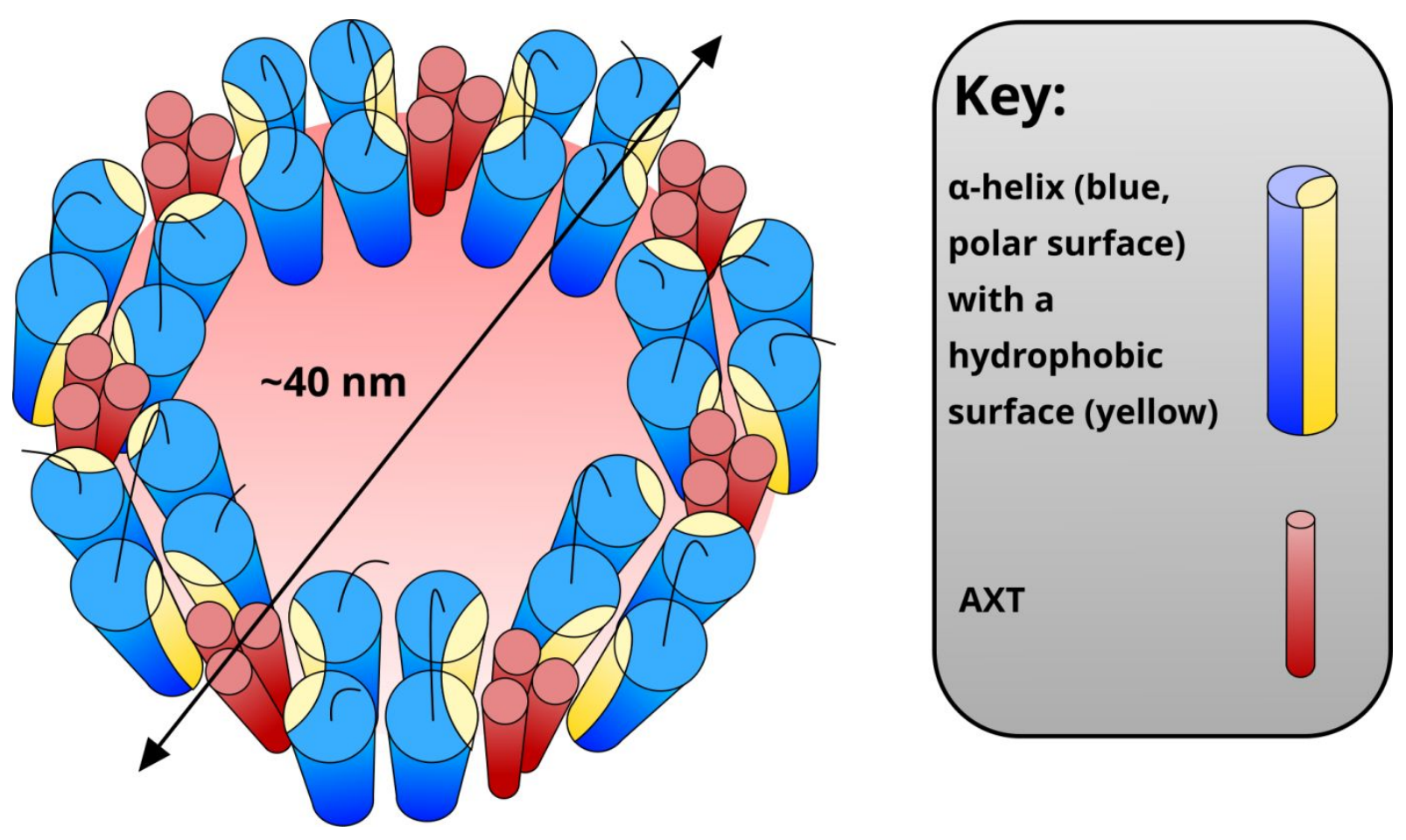

Figure S13. Illustrative model of a 4A-AXT nanoparticle. Individual proteins are represented by four, linked blue cylinders and AXT by smaller red cylinders; cartoon representations approximately to size. Protein surface hydrophobicity is represented in orange; internal hydrophobic patches are omitted for clarity. 


\section{References}

(1) Sutherland, G. A.; Grayson, K. J.; Adams, N. B. P.; Mermans, D. M. J.; Jones, A. S.; Robertson, A. J.; Auman, D. B.; Brindley, A. A.; Sterpone, F.; Tuffery, P.; Derreumaux, P.; Dutton, P. L.; Robinson, C.; Hitchcock, A. Hunter, N. C. Probing the Quality Control Mechanism of the Escherichia Coli Twin-Arginine Translocase with Folding Variants of a De Novo- Designed Heme Protein. J. Biol. Chem. 2018, 293 (18), 6672-6681.

(2) Musser, A. J.; Maiuri, M.; Brida, D.; Cerullo, G.; Friend, R. H.; Clark, J. The Nature of Singlet Exciton Fission in Carotenoid Aggregates. J. Am. Chem. Soc. 2015, 137 (15), 5130-5139.

(3) Kim, H. Y.; Bjorklund, T. G.; Lim, S.-H.; Bardeen, C. J. Spectroscopic and Photocatalytic Properties of Organic Tetracene Nanoparticles in Aqueous Solution. Langmuir 2003, 19 (9), 3941-3946.

(4) Keum, C.-M.; Liu, S.; Al-Shadeedi, A.; Kaphle, V.; Callens, M. K.; Han, L.; Neyts, K.; Zhao, H.; Gather, M. C.; Bunge, S. D.; Twieg R. J.; Jakli, A.; Lüssem, B. Tuning Charge Carrier Transport and Optical Birefringence in Liquid-Crystalline Thin Films: A New Design Space for Organic Light-Emitting Diodes. Sci. Rep. 2018, 8 (1), 699.

(5) Kennehan, E. R.; Grieco, C.; Brigeman, A. N.; Doucette, G. S.; Rimshaw, A.; Bisgaier, K.; Giebink, N. C.; Asbury, J. B. Using Molecular Vibrations to Probe Exciton Delocalization in Films of Perylene Diimides with Ultrafast Mid-IR Spectroscopy. Phys. Chem. Chem. Phys. 2017, 19 (36), 24829-24839.

(6) Hodges, M. P. P.; Grell, M.; Morley, N. A.; Allwood, D. A. Wide Field Magnetic Luminescence Imaging. Adv. Funct. Mater. 2017, 27 (31), 1-7. 